\title{
Effect of Dose and Schedule of L-Asparaginase Administration on Early Minimal Residual Disease in Acute Lymphoblastic Leukemia
}

\begin{abstract}
Background and Objectives: L-asparaginase has become the backbone of acute lymphoblastic leukemia induction. In Berlin-Frankfurt-Munster (BFM) 95/2000 protocols, L-asparaginase was given twice weekly for initial 4 weeks. While sufficient L-asparaginase levels are important, there is no apparent correlation between high L-asparaginase levels and minimal residual disease (MRD). In view of toxicities of L-asparaginase, we planned to study the effect of dose and schedule of Escherichia coli-derived L-asparaginase on early MRD by phasing the same total dose, once a week over 8 weeks. Methods: This prospective, observational study enrolled 45 children and young adults $\leq 40$ years. Modified BFM 95 protocol was followed. Weekly $5000 \mathrm{IU} / \mathrm{m}^{2} \mathrm{~L}$-asparaginase was given intravenously, and MRD was analyzed at the end of 4 weeks (MRD1) and at 8 weeks (MRD2), using multicolor flow cytometry. MRD positive was defined as residual blasts $\geq 0.01 \%$. Results: Thirty-one patients were eligible for final analysis. Nine could receive scheduled eight doses of L-asparaginase and 22 patients received less than eight doses. We analyzed age, gender, diagnosis, prednisone response, cytogenetics, central nervous system status, BFM risk group, MRD2, and relapse. L-asparaginase dose association was not statistically significant with respect to MRD2 $(P=0.237)$. There were no cases of pancreatitis, hypersensitivity, bleeding, or thrombosis. Reasons for patients receiving less than the scheduled eight doses were low serum fibrinogen levels and liver dysfunction. This study revealed 8 MRD1-negative and 13 MRD2-negative patients. Conclusion: L-asparaginase dose intensity does not affect early MRD. Phasing L-asparaginase over 8 weeks could lead to the achievement of more MRD2-negative status and thereby improve long-term outcome. This strategy may also reduce the incidence of adverse drug events.
\end{abstract}

Keywords: Acute lymphoblastic leukemia, Berlin-Frankfurt-Munster, flow cytometry,

L-asparaginase, minimal residual disease

\section{Introduction}

Acute lymphoblastic leukemia (ALL) is a heterogeneous hematologic disease characterized by the proliferation of immature lymphoid cells in the bone marrow, peripheral blood, and other organs with peak incidence in between 2 and 5 years of age. Survival in pediatric ALL has improved to roughly $90 \%$ in trials with risk stratification by biological features of leukemic cells and response to treatment, treatment modification based on patients' pharmacodynamics and pharmacogenomics, and improved supportive care. ${ }^{[1]}$

Minimal residual disease (MRD) in ALL refers to the presence of leukemic cells below the threshold of detection using conventional morphologic methods. Patients who experienced a Complete

This is an open access journal, and articles are distributed under the terms of the Creative Commons Attribution-NonCommercial-ShareAlike 4.0 License, which allows others to remix, tweak, and build upon the work non-commercially, as long as appropriate credit is given and the new creations are licensed under the identical terms.

For reprints contact: reprints@medknow.com
Remission (CR) according to morphologic assessment alone can potentially harbor a large number of leukemic cells in the bone marrow: up to 1010 malignant cells. The most frequently used methods for MRD assessment include multicolor flow cytometry (FCM) to detect abnormal immune phenotypes and polymerase chain reaction (PCR) assays to detect clonal rearrangements in immunoglobulin heavy chain genes and/or T-cell receptor genes. Current FCM or PCR methods can detect leukemic cells at a sensitivity threshold of fewer than $1 \times 10^{-4}(<0.01 \%)$ bone marrow mononuclear cells. The concordance rate for detecting MRD between these methods is high.

The clinical significance of MRD has been conclusively demonstrated in both childhood and adult ALLs. In most studies, MRD positivity is defined by

How to cite this article: Gupta S, Sehrawat A, Dang K, Sharma L, Jaiswal SR, Chakarbarti S. Effect of dose and schedule of L-asparaginase administration on early minimal residual disease in acute lymphoblastic leukemia. Indian J Med Paediatr Oncol 2019;40:496-500.

\section{Satyanker Gupta ${ }^{1}$, Amit Sehrawat ${ }^{2}$, Ketan Dang ${ }^{3}$, Lalit Sharma ${ }^{2}$, Sarita R. Jaiswal ${ }^{4}$, Suparno Chakarbarti $^{4}$}

${ }^{I}$ Department of Medical Oncology, Shri Mata Vaishno Devi Narayana Superspeciality Hospital, Katra, Jammu and Kashmir, ${ }^{2}$ Departments of Medical Oncology, ${ }^{4} B M T$ and Hematology, Dharamshila Narayana Superspeciality Hospital, ${ }^{3}$ Department of Medical Oncology, Max Superspeciality Hospital Saket, New Delhi, India

Submitted: 05-May-2018 Accepted in Revised Form: 21-Jun-2018

Published: 17-Feb-2020

Address for correspondence:

Dr. Satyanker Gupta, Shri Mata Vaishno Devi Narayana Superspeciality Hospital, Katra - 182320 , Jammu and Kashmir, India. E-mail: guptasatyanker@gmail. com

Access this article online Website: www.ijmpo.org DOI: 10.4103/ijmpo.ijmpo_106_18 Quick Response Code:

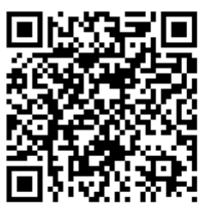


the presence of $0.01 \%$ or more ALL cells; the risk of relapse is generally proportional to the level of MRD, particularly when measured during or at the end of remission induction therapy. The prevalence of MRD during early therapy differs among genetic and biologic ALL subtypes. However, being a measurement of drug resistance in vivo and reflecting multiple cellular, host, and treatment variables, MRD is typically an independent prognostic factor. MRD is now used in several clinical trials for risk assignment and to guide clinical management overall. ${ }^{[2,3]}$

Owing to the unique anticancer mechanism of action, L-asparaginase has been introduced to the multidrug chemotherapy in children and adults with ALL, which has contributed to significant improvement of therapy outcomes and to achieve complete remission in about $90 \%$ of patients. L-asparaginase acts by reducing the exogenous supply of asparagine by catalyzing its hydrolysis, inducing a relative deficiency of asparagine which leads to apoptosis and impaired protein synthesis in leukemic blasts. Notwithstanding its high therapeutic efficacy, L-asparaginase can increase the risk of thrombosis and other complications. ${ }^{[4]}$ Previous studies have proposed that enzyme activity $>100 \mathrm{U} / \mathrm{L}$ ensures complete asparagine depletion. ${ }^{[5]}$

In Berlin-Frankfurt-Munster (BFM) 95/2000 protocols, Escherichia coli-derived L-asparaginase was given twice weekly for initial 4 weeks of induction therapy. Within the ALL BFM 2000 trial, therapeutic drug monitoring of L-asparaginase done on day +3 after administering $5000 \mathrm{IU} / \mathrm{m}^{2}$ of $E$. coli-derived L-asparaginase was compared with MRD on day +33 . The authors found no significant relationship between MRD positivity and differences in L-asparaginase levels. On the contrary, L-asparaginase activity values measured in patients with severe toxicities significantly exceeded those measured in samples from patients without toxicity. ${ }^{[6]}$ In light of above and the severe toxicities encountered with L-asparaginase, especially when co-administered with steroids during induction phase, we planned to study the effect of the standard dose of $E$. coli-derived L-asparaginase on early MRD disease by phasing the same total dose to be given once a week over 8 weeks and study its impact on early MRD at the completion of 4 weeks and then again after the completion of 8 weeks of induction chemotherapy.

\section{Methods}

This study was carried out from February 1, 2014, to April 30, 2016. This study was approved by the Institutional Scientific and Ethical Committee. Informed and written consent was taken from the patients and/or their parents/guardians; in case, the patients were $<18$ years. It was a prospective, observational, pilot study. Forty-five ALL children and young adults up to the age of 40 years were enrolled.
Fourteen patients defaulted during induction treatment and were not eligible for further analysis. Thus, 31 cases were eligible for final analysis. Partially or previously treated, HIV-positive patients allergic to L-asparaginase and those with serum bilirubin $>2 \mathrm{mg} / \mathrm{dl}$, serum glutamic-pyruvic transaminase $>3$ times ULN, creatinine clearance $<50 \mathrm{ml} / \mathrm{min}$, pancreatitis, and serum fibrinogen $<100 \mathrm{mg} / \mathrm{dl}$ were excluded from the study. MRD was assessed using Navios flow cytometer from Beckman Coulter. Six-color FCM analysis was done using fluorescein isothiocyanate, phycoerythrin (PE), allophycocyanin, PE-Cy5, PE-Cy7, and electron-coupled dye.

Patients were subjected to complete systemic examination, assessment of performance status, body surface area and central nervous system (CNS). Investigations done included complete blood counts, liver \& renal function tests, tumor lysis profile, serum fibrinogen levels, bone marrow aspiration and biopsy, FCM from aspiration sample, cerebrospinal fluid analysis and 2D Echocardiography. After diagnosing the patients, the patients were started on preinduction with prednisone along with the first dose of intrathecal methotrexate. Patients were treated according to modified BFM 95 protocol [Table 1a and b].

MRD1 assessment was done at the end of 4 weeks, i.e., day 36 and MRD2 at the end of Phase B, i.e., day 72. Up to 1 million events were studied. CD45 blast gating method was used to discriminate leukemic blasts from the cells of various lineages and to facilitate the analysis of leukemic blasts present at low frequencies. MRD positive was defined as residual blasts $\geq 0.01 \%$ whereas MRD negative was labeled as $<0.01 \% .{ }^{[2]}$ Patients were evaluated with liver function test, amylase, and fibrinogen thrice a week during Phase A and once a week during Phase B.

For statistical analysis of MRD at the end of 4 weeks and 8 weeks, we applied student's $t$-test (Levene's test of

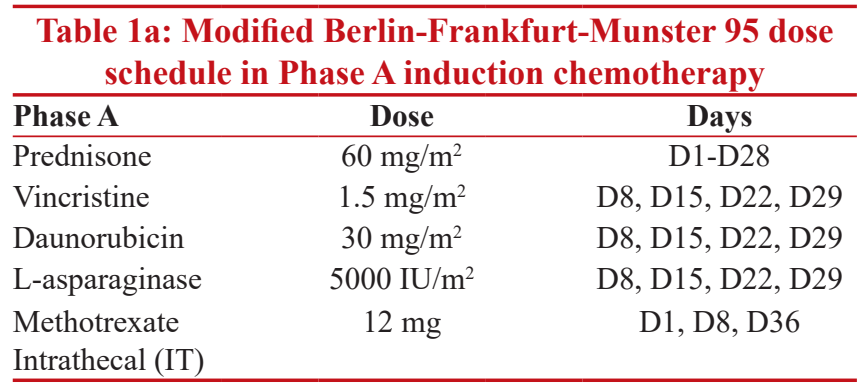

Table 1b: Modified Berlin-Frankfurt-Munster 95 dose schedule in Phase B induction chemotherapy

\begin{tabular}{lcc}
\hline Phase B (INJ) & Dose & Days \\
\hline Cyclophosphamide & $1000 \mathrm{mg} / \mathrm{m}^{2}$ & D1 and D22 \\
Cytarabine & $75 \mathrm{mg} / \mathrm{m}^{2}$ & D1, D8, D15, D22 \\
L-asparaginase & $5000 \mathrm{IU} / \mathrm{m}^{2}$ & D1, D8, D15, D22 \\
Methotrexate IT & $12 \mathrm{mg}$ & D1, D22 \\
\hline D - Day; INJ - Injection; IT - Intrathecal; IU - International Units
\end{tabular}


equality of variance) for continuous variables. For binary variables, we applied Chi-square test (Fisher's exact test).

\section{Results}

The patient characteristics at baseline are shown in Table 2 and Figure 1. The median age of the patients diagnosed with ALL was 14 years (range, 2-40), both females and males were diagnosed, and males were found to be more in number than females. In this study, 22 cases were B-cell immune phenotype and the remaining 9 cases belonged to T-cell immunophenotype. No case of testicular involvement was seen. The BFM 95 risk group stratified 7, 16, and 8 patients as having standard risk, medium risk, and high risk (HR), respectively. Good prednisolone response was observed on day 8 in 23 participants as compared to poor prednisolone response which was seen in 8 cases.

\begin{tabular}{|c|c|}
\hline & $n$ \\
\hline \multicolumn{2}{|l|}{ Age (years) } \\
\hline $1-10$ & 13 \\
\hline$>10-40$ & 18 \\
\hline \multicolumn{2}{|l|}{ Gender } \\
\hline Female & 8 \\
\hline Male & 23 \\
\hline \multicolumn{2}{|l|}{ Diagnosis } \\
\hline B-cell ALL & 22 \\
\hline T-cell ALL & 9 \\
\hline \multicolumn{2}{|l|}{ Risk group } \\
\hline SR & 7 \\
\hline MR & 16 \\
\hline HR & 8 \\
\hline \multicolumn{2}{|l|}{ Prednisolone response } \\
\hline GPR & 23 \\
\hline PPR & 8 \\
\hline \multicolumn{2}{|l|}{ CNS status } \\
\hline CNS 1 & 29 \\
\hline CNS 2 & 1 \\
\hline CNS 3 & 1 \\
\hline \multicolumn{2}{|l|}{ Cytogenetics } \\
\hline High hyperdiploidy & 2 \\
\hline Normal karyotype & 24 \\
\hline HR karyotype & 5 \\
\hline \multicolumn{2}{|l|}{ L-asparaginase dose } \\
\hline$<8$ doses & 22 \\
\hline 8 doses & 9 \\
\hline \multicolumn{2}{|l|}{ MRD1 values } \\
\hline MRD negative & 8 \\
\hline MRD positive & 23 \\
\hline \multicolumn{2}{|l|}{ MRD2 values } \\
\hline MRD negative & 13 \\
\hline MRD positive & 18 \\
\hline
\end{tabular}

The CNS status evaluation as per the BFM 95 protocol at diagnosis categorized 29 cases with CNS Status 1 whereas CNS Status 2 and 3 was diagnosed in one patient each. The cytogenetic evaluation revealed two patients as high hyperdiploidy and 5 participants as having HR cytogenetics. The remaining 24 participants had normal karyotype [Table 2].

The L-asparaginase dose was dichotomized into those who received 8 doses and the others receiving less than eight doses are shown in Table 3.

The median dose of L-asparaginase received during Phase $\mathrm{A}$ and $\mathrm{B}$ was 4 (range, 1-4). However, the total median dose was 7 (range, 2-8). The main reason behind patients receiving less than the scheduled eight doses was low serum fibrinogen levels and liver dysfunction.

There were no cases of pancreatitis, thrombosis, or hypersensitivity. Our results showed that L-asparaginase dose was not statistically significant with respect to MRD2 $(P=0.237)$. The median L-asparaginase dose received in this study was 7 , which was also not statistically significant with respect to MRD2.

\section{Discussion}

Owing to the unique anticancer mechanism of action, L-asparaginase has been introduced to the multidrug chemotherapy in children and adults with ALL, which has contributed to significant improvement of therapy outcomes and to achieve complete remission in about $90 \%$ of patients. ${ }^{[4,7,8]}$ BFM-inspired protocols continue to be the most commonly followed treatment for patients with ALL and L-asparaginase remains a crucial block in the treatment protocols. However, most of the patients were unable to receive the prescribed doses of L-asparaginase within a short span either due to derangements in the metabolic parameters such as low serum fibrinogen levels, deranged liver function tests, thrombotic events, or due to severe allergy/hypersensitivity.

There were no cases of drug hypersensitivity to L-asparaginase, pancreatitis, or bleeding in this study.

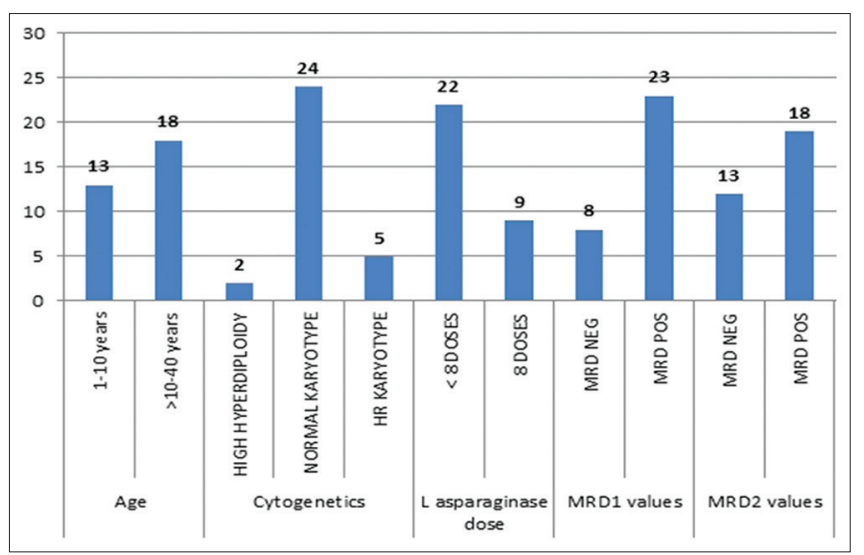

Figure 1: Study population distribution 


\begin{tabular}{|c|c|c|c|}
\hline & & & \\
\hline \multirow{2}{*}{\multicolumn{4}{|c|}{ Gender }} \\
\hline & & & \\
\hline Male & 7 & 16 & \multirow{2}{*}{$\begin{array}{l}\text { Fisher's exact test } \\
\text { (two-sided) } 1.00\end{array}$} \\
\hline Female & 2 & 6 & \\
\hline \multicolumn{4}{|l|}{ Diagnosis } \\
\hline B cell & 7 & 15 & \multirow{2}{*}{$\begin{array}{l}\text { Fisher's exact test } \\
\text { (two-sided) } 0.689\end{array}$} \\
\hline $\mathrm{T}$ cell & 2 & 7 & \\
\hline \multicolumn{4}{|l|}{ Prednisolone response } \\
\hline GPR & 7 & 16 & \multirow{2}{*}{$\begin{array}{l}\text { Fisher's exact test } \\
\text { (two-sided) } 1.00\end{array}$} \\
\hline PPR & 2 & 6 & \\
\hline \multicolumn{4}{|l|}{ Cytogenetics } \\
\hline High hyperdiploidy & 1 & 1 & \multirow{3}{*}{$\begin{array}{l}\text { Fisher's exact test } \\
\text { (two-sided) } 0.635\end{array}$} \\
\hline Normal karyotype & 17 & 6 & \\
\hline High risk karyotype & 3 & 2 & \\
\hline \multicolumn{4}{|l|}{$\mathrm{CNS}$} \\
\hline 1 & 9 & 20 & \multirow{3}{*}{$\begin{array}{l}\text { Fisher's exact test } \\
\text { two-sided) } 1.00\end{array}$} \\
\hline 2 & 0 & 1 & \\
\hline 3 & 0 & 1 & \\
\hline \multicolumn{4}{|l|}{ BFM risk group } \\
\hline SR & 4 & 3 & \multirow{3}{*}{$\begin{array}{l}\text { Fisher's exact test } \\
\text { (two-sided) } 0.189\end{array}$} \\
\hline MR & 4 & 12 & \\
\hline HR & 1 & 7 & \\
\hline \multicolumn{4}{|l|}{ MRD 2} \\
\hline Positive & 7 & 11 & \multirow{2}{*}{$\begin{array}{l}\text { Fisher's exact test } \\
\text { (two-sided) } 0.237\end{array}$} \\
\hline Negative & 2 & 11 & \\
\hline
\end{tabular}

SR - Standard risk; MR - Medium risk; HR - High risk; GPR - Good prednisolone response; PPR - Poor prednisolone response;

MRD - Minimum residual disease; BFM - Berlin-Frankfurt-Munster; CNS - Central nervous system

There were no cases of thrombosis in this study whereas coagulopathy was reported among $2.1-15 \%$ and up to $30.2 \%$ of patients in the comparable studies. ${ }^{[4,9]}$

Our results clearly demonstrate that there is no correlation between L-asparaginase dose intensity and MRD levels obtained at the end of induction treatment. These results are in concordance with the results derived from the therapeutic drug monitoring study in BFM 2000 trial. $^{[6]}$

The results of this study suggest that the scheduled eight doses of L-asparaginase can be safely given over 8 weeks instead of giving all the doses during Phase $\mathrm{A}$ as per the BFM 95 protocol. In this study, 13 patients were MRD2 negative as compared to only eight patients who were MRD1 negative, and none of these MRD-negative patients relapsed. This happened probably due to the additional doses of L-asparaginase received during Phase $\mathrm{B}$ induction therapy. It has been well documented in the previous studies that achievement of MRD-negative status at the end of induction chemotherapy correlates with long-term outcome in ALL. The detection of MRD is a major prognostic factor for treatment in ALL of childhood. Several groups showed the predictive value of MRD at the end of induction therapy with low MRD values correlating with better long-term outcome. ${ }^{[10,11]}$

This study demonstrates the feasibility of administering 8 doses of L-asparaginase over two phases rather than over the 4-week period as per the original BFM protocols. This was associated with reduced toxicity and did not compromise the efficacy of the therapy.

\section{Conclusion}

L-asparaginase is the backbone of induction therapy in ALL for decades. The dosing schedules vary across all the standard protocols. L-asparaginase dose intensity does not affect early MRD (neither MRD1 nor MRD2). Phasing L-asparaginase over 8 weeks could lead to the achievement of more MRD2-negative patients and thereby positively impacting their long-term outcome. Phasing of L asparaginase over 8 weeks may result in reduced incidence of adverse drug events.

\section{Financial support and sponsorship}

Nil.

\section{Conflicts of interest}

There are no conflicts of interest.

\section{References}

1. Inaba $\mathrm{H}$, Greaves $\mathrm{M}$, Mullighan CG. Acute lymphoblastic leukaemia. Lancet 2013;381:1943-55.

2. Campana D. Minimal residual disease in acute lymphoblastic leukemia. Hematology Am Soc Hematol Educ Program 2010;2010:7-12. 
3. Bartram CR, Schrauder A, Köhler R, Schrappe M. Acute lymphoblastic leukemia in children: Treatment planning via minimal residual disease assessment. Dtsch Arztebl Int 2012;109:652-8

4. Piatkowska-Jakubas B, Krawczyk-Kuliś M, Giebel S, Adamczyk-Cioch M, Czyz A, Lech Marańda E, et al. Use of L-asparaginase in acute lymphoblastic leukemia: Recommendations of the polish adult leukemia group. Pol Arch Med Wewn 2008;118:664-9.

5. Riccardi R, Holcenberg JS, Glaubiger DL, Wood JH, Poplack DG. L-asparaginase pharmacokinetics and asparagine levels in cerebrospinal fluid of rhesus monkeys and humans. Cancer Res 1981;41:4554-8.

6. Schrey D, Borghorst S, Lanvers-Kaminsky C, Hempel G, Gerss J, Möricke A, et al. Therapeutic drug monitoring of asparaginase in the ALL-BFM 2000 protocol between 2000 and 2007. Pediatr Blood Cancer 2010;54:952-8.

7. Nesbit ME, Ertel I, Hammond GD. L-asparaginase as a single agent in acute lymphocytic leukemia: Survey of studies form childrens cancer study group. Cancer Treat Rep 1981;65 Suppl 4:101-7.

8. Chessells JM, Richards SM, Bailey CC, Lilleyman JS, Eden OB. Gender and treatment outcome in childhood lymphoblastic leukaemia: Report from the MRC UKALL trials. Br J Haematol 1995;89:364-72.

9. Pession A, Valsecchi MG, Masera G, Kamps WA, Magyarosy E, Rizzari C, et al. Long-term results of a randomized trial on extended use of high dose L-asparaginase for standard risk childhood acute lymphoblastic leukemia. J Clin Oncol 2005;23:7161-7.

10. Stow P, Key L, Chen X, Pan Q, Neale GA, Coustan-Smith E, et al. Clinical significance of low levels of minimal residual disease at the end of remission induction therapy in childhood acute lymphoblastic leukemia. Blood 2010;115:4657-63.

11. Samra MA, Mahmoud HK, Abdelhamid TM, El Sharkawy NM, Elnahass YH, Elgammal M, et al. The prognostic significance of minimal residual disease in adult Egyptian patients with precursor acute lymphoblastic leukemia. J Egypt Natl Canc Inst $2013 ; 25: 135-42$ 